\title{
Computer-based Simulation of the Wayamba Unmanned Underwater Vehicle
}

\author{
C. Madden ${ }^{\text {a }}$, D. Sgarioto ${ }^{\text {a }}$ \\ ${ }^{a}$ Maritime Division, Defence Science and Technology Organisation, Melbourne, Australia \\ Email: daniel.sgarioto@dsto.defence.gov.au
}

\begin{abstract}
Unmanned systems continue to generate significant interest from a wide range of entities, including researchers, vehicle operators and the general public. Compared to legacy manned platforms, unmanned systems have the potential to provide increased and persistent situational awareness whilst exposing operators to less risk. Although unmanned systems may provide novel capabilities, they often require significant manpower to operate and can also be expensive to develop, operate and sustain. Computer-based modelling and simulation provides a cost effective means to develop and evaluate appropriate designs and the concepts of operations that underlie the proficient tactical use of unmanned systems. It is paramount that accurate models are utilised to ensure simulations produce meaningful results, so that designs can be adapted as early as possible to reduce costs. Moreover, to reduce complexity and lead times of simulation development, developers can exploit an increasing number of existing tools. These include Commercial Off The Shelf (COTS) software to assist with the development of motion models, though the abstraction of such tools may limit the physical understanding of the vehicle that may be gained from deriving equations of motion from first principles. State-of-the-art virtual environment technologies allow for the creation of realistic modelling environments, in which numerous vehicle motion and sensor models can be integrated within a single simulation facility.
\end{abstract}

This paper outlines the results of research undertaken at the Defence Science and Technology Organisation (DSTO) towards the development of a computer-based simulation facility for the Wayamba Unmanned Underwater Vehicle (UUV) project. The simulation suite includes a higher level COTS UUV simulation system called AUVSim that can be used as a virtual environment for Hardware-In-the-Loop (HIL) simulations, and a DSTO-developed, MATLAB ${ }^{\circledR}$-based model of the vehicle, for investigating low-level vehicle dynamics and performance. The low-level vehicle model was formulated using the "component build up method" and does not require the determination of hydrodynamic derivatives, reducing both the development time and underlying complexity of the model. Its derivation involves decomposing the vehicle into its basic elements, including the hull, conning tower, bowplane control surfaces, fixed sternplanes and the propulsion system represented by a pair of aft thrusters. At this stage, the model does not incorporate the vehicle's through body tunnel thrusters. The AUVSim vehicle model is constructed using a similar "component build up method", but the underlying equations of motion that govern the resulting vehicle model are not accessible to the user. This COTS "black box" simulation model has a shorter development time than the low-level model and is best suited to investigating the effect of vehicle hardware configuration changes and their influence on the vehicle's ability to conduct various manoeuvres. The low-level vehicle model is fully adaptable and can be used to investigate a wider range of operating conditions for the vehicle, including new actuator types. The representativeness of both the low-level and COTS model is established by comparing relevant simulation results with real vehicle data obtained from field trials. To facilitate further development and improve the fidelity of these simulation models, outstanding areas of research and directions for future work are also provided.

Keywords: Unmanned underwater vehicles, modelling, simulation, autonomy 


\section{INTRODUCTION}

Operating vehicles in the dynamic maritime environment provides a range of unique opportunities and challenges. These are amplified when the vehicle is operated as an unmanned system where it is either remotely operated, or controlled by an autonomous system. Such systems have a range of novel capabilities, such as improved stealth, and increased and persistent situational awareness, whilst exposing personnel to lower risk.

Currently these systems still require significant manpower to operate the vehicles, or intervene if the vehicle is not behaving as expected. This occurs because the autonomous control systems are often not trusted to conduct their tasks and respond to the developing situation appropriately. This is heightened as many of the advanced systems are currently developed as research platforms and may suffer from a higher degree of hardware and software problems than commercial models. Developing appropriate simulation environments allows for vehicles to be tested more frequently, safely and cost effectively as it can reduce the number of trials required to become confident in the system's operation.

\subsection{Background}

The 2013 Defence White Paper, Australian Department of Defence (2013), highlights Australia's continued focus on developing its capability to manage its large maritime environment. Whilst it focuses upon future manned platforms, there is also an increasing interest within the Royal Australian Navy (RAN) in using unmanned vehicles to increase its operational capability and flexibility. One possibility includes the use of modular unmanned systems that can be applied to adapt a ship to a range of tasks, such as that used in the American Littoral Combat Ship (LCS) as outlined in Schnoor (2003). To effectively develop such capability it is important to be able to generate models and simulations of critical system components early, in order to properly evaluate the capabilities they can provide. To run sea trials examining such capabilities with a wide range of assets can often be programmatically difficult and prohibitively expensive to organise the required assets, including system prototypes, and may also have significant risks, especially with appropriate weather being required during the trial's time frame.

Developing appropriate simulations of the unmanned system concepts can therefore be very important to identify and overcome any design problems early, especially when the simulations can be combined to evaluate a vehicle or system's full range of capabilities. Whilst it is useful to simulate systems which are yet to be built, it is important to ensure the simulation reflects reality. To verify the simulation infrastructure produces realistic results, it is important to compare the results of simulations with data obtained from real systems, such as data obtained from the operation of DSTO's Wayamba UUV.

\subsection{Wayamba, DSTO's Unmanned Underwater Vehicle}

Since 1998, DSTO has been examining UUV technology through the development of the novel Wayamba research vehicle. An initial report detailing the original design and performance specifications for Wayamba can be found in Coxhead et al. (2002). The Wayamba platform is a tethered vehicle with a flatfish design which is now approximately $4 \mathrm{~m}$ long, weighs approximately $1400 \mathrm{~kg}$ and has a maximum speed of approximately $1.8 \mathrm{~m} / \mathrm{s}$. Its main propulsion consists of 2 large rear propellers [Port Main (PM) and Starboard Main (SM)], with its high degree of manoeuvrability provided by a combination of 2 bowplanes [Port Bowplane (PB) and Starboard Bowplane (SB)] and 3 ducted vertical thrusters [Front Vertical (FV), Port Vertical (PV) and Starboard Vertical (SV)] and one ducted lateral thruster [Rear Lateral (RL)], as shown in Figure 1. This combination is currently powered through its umbilical tether, which also provides a fibre optic data link to the vehicle's internal network, including the onboard computer system. The vehicle's high degree of flexibility in its remote operation facilitates system-level testing and the integration of components for investigating its increasing level of automation. The vehicle's control system consists of either manual remote control, which has the vehicle actuators mapped to an Xbox controller, or a semi-autonomous autopilot that uses defined set points for vehicle control, such as maintaining speed, heading, and depth, as well as path following via waypoint navigation. Madden et al. (2012) provides an overview of the integration of the autopilot into the vehicle's control system, including the results of initial sea trials.

\section{SIMULATING UNMANNED UNDERWATER VEHICLES}

The development, operation and sustainment of UUV technology is a complex, lengthy and expensive undertaking. Operating UUVs also has significant risks, most notably vehicle loss, fixed costs associated with support personnel, logistics, equipment, and facilities, which can be prohibitively expensive. As a result, devel- 

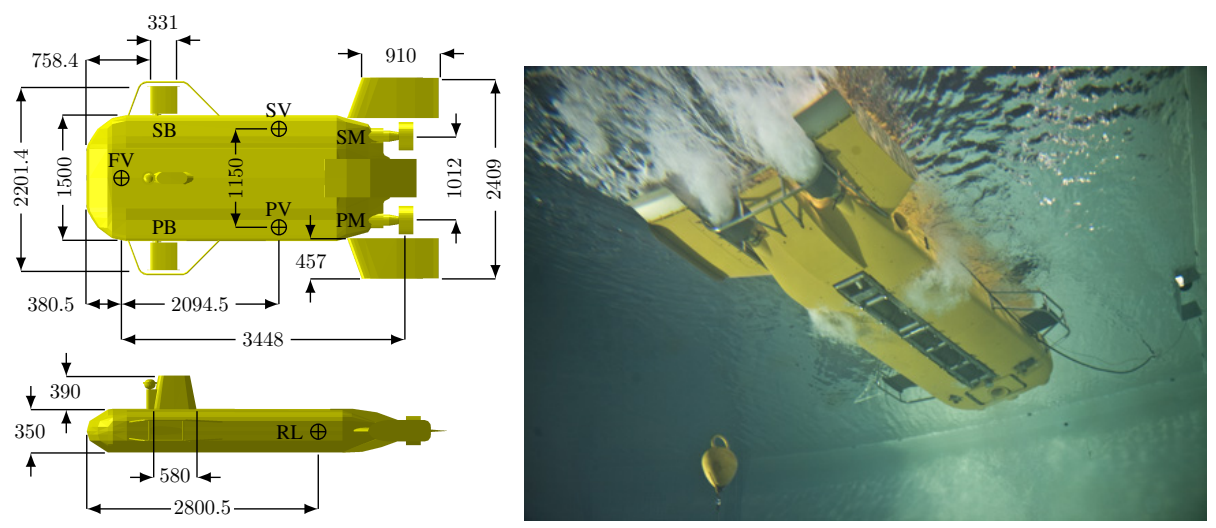

Figure 1. Schematic of the Wayamba UUV and it operating in DSTO's water tank

opers and operators of UUVs are increasingly utilising computer-based simulation, to both identify potential operational capabilities and optimise concepts of operations, all without risking the actual vehicle. A major challenge when creating such a simulation capability is verifying that representative models are developed to ensure simulations produce meaningful results.

An underwater vehicle fully submerged in an unbounded flow field possesses six degrees-of-freedom (DOF), three corresponding to translational motion along three perpendicular axes and the remaining three relating to rotational motion about these same axes. In order to properly classify the dynamic motion of an underwater vehicle, it is convenient to utilize the two coordinate systems shown in Figure 2.

One such coordinate system is a global inertial reference frame $X Y Z$, which comprises the underwater environment. This right-handed reference frame is defined by the unit vectors in the $X$ direction (North), $Y$ direction (East) and $Z$ direction (Down) respectively. The other coordinate system is a right-handed moving reference frame $x y z$ fixed to the vehicle's centre of buoyancy. The origin $O$ of the body-fixed frame is chosen so that the respective axes coincide with the principal axes of inertia for the vehicle. The local body reference frame is characterised by the unit vectors in the $x$ direction (from aft to fore), $y$ direction (starboard) and $z$ direction (from top to bottom) respectively.

The position and orientation of an underwater vehicle are referenced relative to the global inertial frame, whilst the linear and angular velocities are expressed in the local body-fixed frame. In accordance with the standard convention outlined in Fossen (1994), the inertial position $\eta_{1}$, the translational velocity $\nu_{1}$, the Euler angles $\eta_{2}$ and the angular rates $\nu_{2}$ for an underwater vehicle are given by:

$$
\eta_{1}=\left\{\begin{array}{c}
X \\
Y \\
Z
\end{array}\right\} \quad \nu_{1}=\left\{\begin{array}{c}
u \\
v \\
w
\end{array}\right\} \quad \eta_{2}=\left\{\begin{array}{c}
\phi \\
\theta \\
\psi
\end{array}\right\} \quad \nu_{2}=\left\{\begin{array}{c}
p \\
q \\
r
\end{array}\right\}
$$

where $u$ is the surge, $v$ is the sway, and $w$ is the heave speed; $\phi$ is the roll, $\theta$ is the pitch, and $\psi$ is the yaw angle; $p$ is the roll rate, $q$ is the pitch rate and $r$ is the yaw rate for the vehicle respectively.
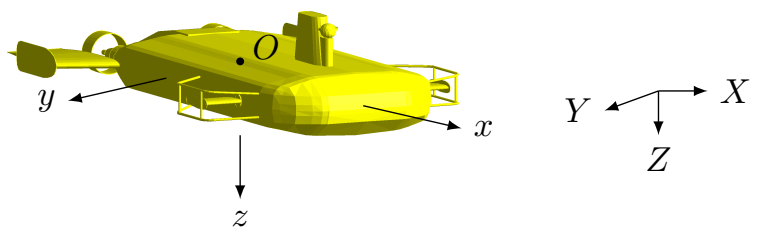

Figure 2. Global inertial and local body-fixed reference frames 


\subsection{DSTO's Low-level Non-linear Vehicle Model}

The DSTO low-level non-linear dynamic model of the Wayamba UUV was synthesised in MATLAB ${ }^{\circledR}$ using the "component build up method" first developed by Nahon (1996) and later refined by Evans and Nahon (2004). Although a variety of modelling and simulation procedures have been proposed for streamlined UUVs, no accepted standard procedure exists, especially for flatfish-type UUVs such as Wayamba. Nahon's approach to modelling UUVs was chosen since it does not require the determination of hydrodynamic derivatives for the vehicle, reducing significantly both the development time and underlying complexity of the resulting vehicle model. Hydrodynamic forces are simply determined from well-known empirical relations which only require the specification of the vehicle's geometry. For a detailed derivation of this procedure, readers should consult the seminal work of Fossen (1994).

The derivation of the low-level vehicle model involves decomposing the vehicle into its basic elements, including the hull, conning tower, bowplane control surfaces, fixed sternplanes and the propulsion system represented by a pair of aft thrusters. Each component is modelled using simple hydrodynamic expressions derived from empirical relations. The forces and moments from each of these components are combined to determine the total hydrodynamic load acting on the vehicle. Any corresponding control forces and moments are also included in the summation process to complete the derivation procedure. Although the resulting model is relatively simple, the vehicle's fundamental nonlinear behaviour is retained.

The low-level vehicle model utilises an autonomous control system for motion stabilisation and manoeuvring. The general approach used to synthesize a controller for the low-level vehicle model is based on the decoupled controller design paradigm, whereby a set of separate designs for speed, roll, steering and diving control are obtained, in line with the conventional UUV control design philosophy outlined in Healey and Lienard (1993). As reported by Madden et al. (2012), the actual vehicle uses field-tuned Proportional Integral Derivative (PID) controllers to control its motion. Hence, the speed control autopilot for low-level vehicle model is a basic Proportional (P) controller using an error signal based on the vehicle's forward speed, with the control input itself being the commanded thrust of the vehicle's rear main thrusters. A Proportional-Derivative (PD)-type controller forms the basis of the vehicle's diving autopilot. This controller ensures the vehicle maintains a particular depth, using only bow plane angle alterations to perform depth-changing manoeuvres as the vehicle's ducted vertical and lateral thrusters are not yet incorporated into the low-level vehicle model. The vehicle's steering autopilot is a simple PD controller used to command the vehicle to attain a given heading by altering the balance of thrust provided by the vehicle's rear main thrusters.

\subsection{Hardware-in-the-loop Simulation Model}

The HIL simulation model was developed using COTS software from H-Scientific (2010). This software uses a similar "component build up method" to the low-level model, although the underlying equations of motion that govern the vehicle model are not accessible to the user. This abstraction reduces both the development time and complexity of the resulting model, but limits the degree of physical insight potentially offered by the model. Based on the layout given in Figure 1, AUVSim uses the position, orientation, and operational characteristics of the vehicle's actuators to create a vehicle simulation model. The rear main thrusters have a radius of $0.14 \mathrm{~m}$, an RPM ceiling of 1000 and an RPM rate limit of 250, whilst the remaining thrusters have a radius of $0.09 \mathrm{~m}$, an RPM ceiling of 1600 and an RPM rate limit of 500. The bowplanes are constrained to have a maximum deflection angle of $15^{\circ}$ and a rate limit of $15^{\circ} \mathrm{deg} / \mathrm{s}$. An additional force, representing the drag on the rear of the vehicle due to its tether cable, is also included in the AUVSim vehicle model.

The manoeuvring of the vehicle can be conducted either by connection to a physical Spectre autopilot unit, such as Wayamba uses, or with the simulated autopilot software built into the AUVSim software. Both methods provide the same software signals to provide the manoeuvring control signals. The simulation provides outputs representing virtual copies of the standard navigation sensors for UUVs, such as a GPS or INS measurements. This allows for the software to provide a virtual motion data set for hardware and software in the loop simulation. The system does not recreate external sensors, such as sonars, and has a basic simulated world environment, which limits the simulation as only the motion of a single vehicle is incorporated.

\section{RESULTS}

This section outlines two demonstrative case study scenarios illustrating the use of the computer-based simulation facility for the Wayamba UUV. The representativeness of this simulation environment is found by comparing both the low-level and hardware-in-the-loop simulation models to actual Wayamba UUV field data 
obtained during two manoeuvres conducted as a part of sea trials at Corio Quay.

\subsection{Simultaneous Speed and Heading Control}

The first proposed case study manoeuvre involved the vehicle decelerating while simultaneously undergoing a medium sized heading alteration. The initial conditions governing this scenario were the equilibrium condition of the vehicle travelling steady and level at a speed of $1.05 \mathrm{~m} / \mathrm{s}$, a depth of $5 \mathrm{~m}$ and an initial heading of $100^{\circ}$ (zero pitch and roll). The goal of each simulation model is to command the vehicle to attain a new heading of $165^{\circ}$ at $0.6 \mathrm{~m} / \mathrm{s}$. Figure 3 and Figure 4 depict the results obtained using each simulation model, together with the actual recorded vehicle data. The results concerning the low-level vehicle model are given by the blue line; the magenta line denotes the AUVSim model results, the green line signifies the results obtained from the real vehicle, whilst the red line indicates the goal to be obtained by each simulation model.
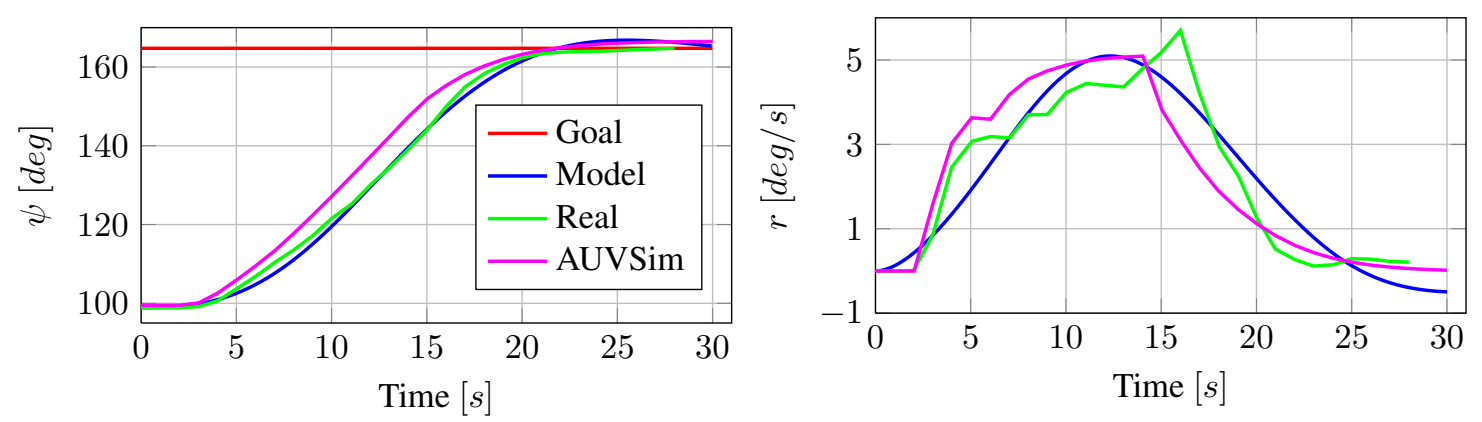

Figure 3. Vehicle Heading and Yaw Rate Time Histories for Manoeuvre 1

Figure 3 shows that each model is successfully able to attain the desired heading goal with minor overshoot. The low-level model tracks the real vehicle's heading response more closely, whilst the AUVSim model performs a more aggressive turning manoeuvre, which is also evident in the yaw (turn) rate time histories obtained using each model. Figure 4 indicates that the trajectories predicted by both simulation models, especially the AUVSim model, closely match the trajectory followed by real vehicle. Figure 4 also reveals that both models successfully reach the desired speed goal. However, the surge speed variations predicted by the AUVSim model are more consistent with those experienced by the real vehicle. The speed controller implemented within the low-level model produces considerable overshoot, a phenomenon not experienced by the real vehicle.
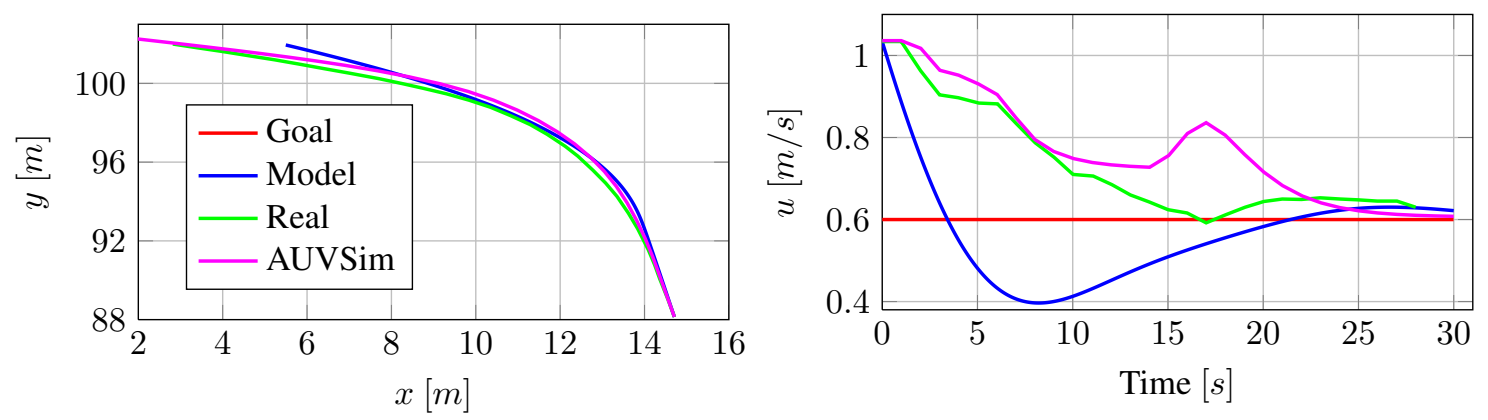

Figure 4. Vehicle Trajectory and Surge Velocity Time Histories for Manoeuvre 1

\subsection{Sequential Speed and Depth Control}

The second proposed case study manoeuvre involves the vehicle accelerating for $20 s$ before changing its depth from $5 \mathrm{~m}$ down to $7 \mathrm{~m}$. The initial conditions governing this scenario was the equilibrium condition of 
the vehicle travelling steady and level at a speed of $0.79 \mathrm{~m} / \mathrm{s}$, a depth of $5 \mathrm{~m}$ and an initial heading of $5^{\circ}$ (zero pitch and roll). The goal of each simulation model is to command the vehicle to attain a new speed of 1.05 $\mathrm{m} / \mathrm{s}$, then dive after $20 \mathrm{~s}$ to a depth of $7 \mathrm{~m}$. Figures 5 and 6 use the same colour conventions as Section 3.1.
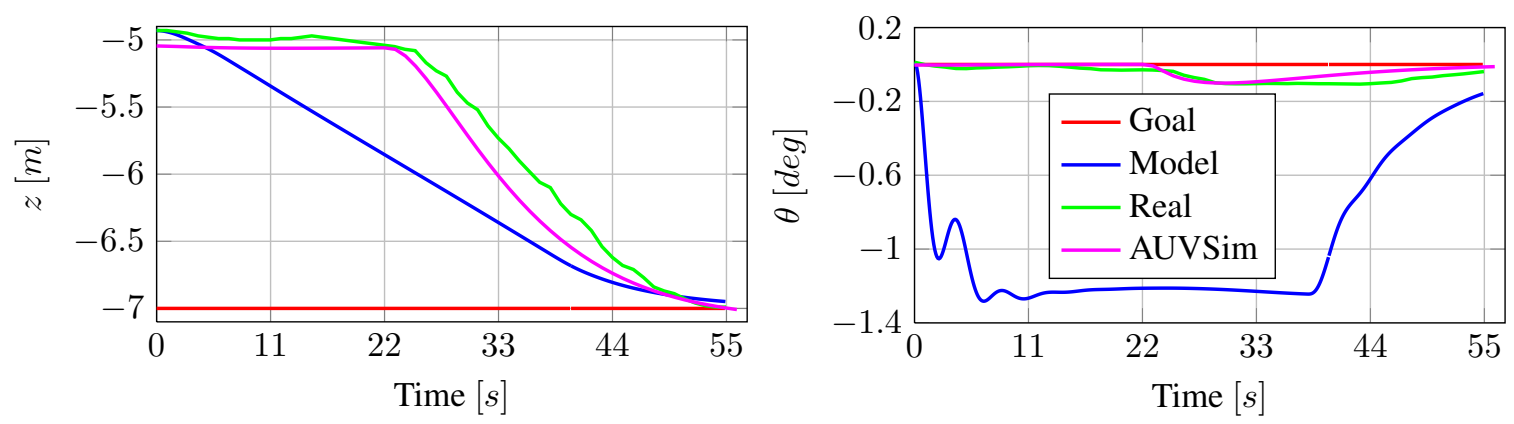

Figure 5. Vehicle Depth and Pitch Angle Time Histories for Manoeuvre 2

Figure 5 shows that each model can successfully achieve the desired depth change, with the AUVSim model more accurately simulating the real vehicle. To achieve the desired goal, the low-level model must perform a different manoeuvre compared to the real vehicle: start diving immediately, with a much larger nose-down pitch angle. This is because, in its current state, the low-level model does not incorporate vertical thrusters, which significantly influence the vehicle's ability to change depth. Figure 6 shows that both the low-level and AUVSim models achieve the velocity change faster than the real vehicle, though the low-level model also overshoots the goal. The AUVSim model appears to slow down at the point where it changes depth, which is not reflected on the real vehicle. The low-level model undergoes significant variation in its heading, which does not occur in either the AUVSim model or the real data. This out-of-plane excitation is due to coupled interactions between the model's controllers, arising from sub-optimal control allocation. In its current form, the applicability of the low-level model is restricted to simulations of the vehicle's lateral $X-Y$ plane motion.
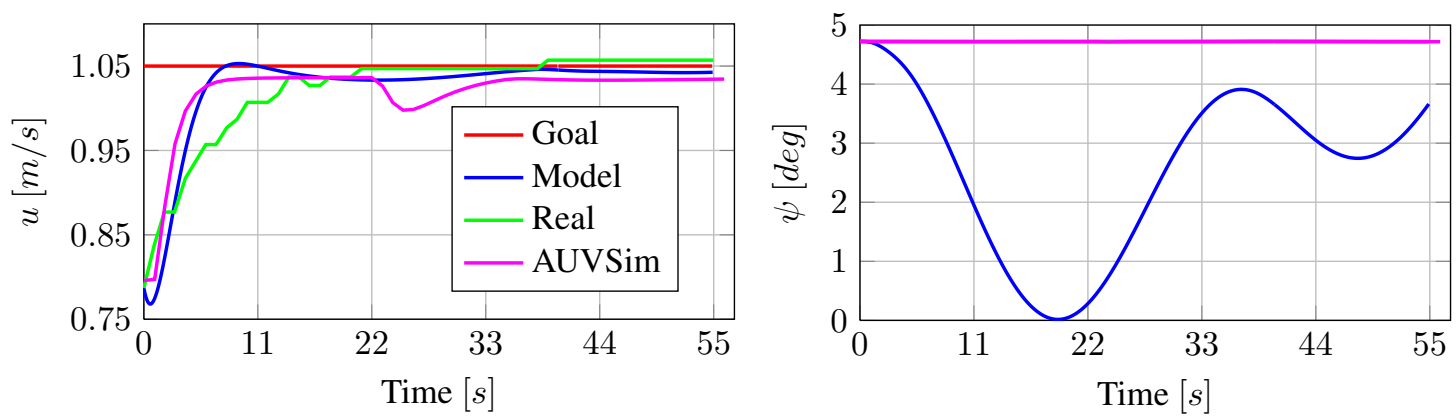

Figure 6. Vehicle Surge Velocity and Heading Time Histories for Manoeuvre 2

\section{FUTURE WORK}

While the results presented in this paper clearly show that the computer-based simulation facility for the Wayamba UUV are representative, there is sufficient scope to further develop this modelling capability. Improved hydrodynamic modelling for the low-level vehicle model is an obvious candidate for future work. Simple expressions were used to determine the main hydrodynamic coefficients of the vehicle. More sophisticated procedures do exist, such as those contained within de Barros et al. (2008), which can account for body-fin interactions and non-linear effects at high angles of attack. In order to ensure that simulations remain 
representative across the total performance envelope of the vehicle, appropriate scheduling of the control gains for the vehicle's autopilot is required.

Work is currently underway to incorporate through-body tunnel thrusters within the low-level vehicle model. This will involve the development of a numerical model for quantifying the effect of speed and orientation angle on the performance of these tunnel thrusters. Modelling the interchange between the tunnel thrusters and the bowplanes during transitions between low and high speed depth-plane manoeuvring is also a future research goal. Control allocation remains a requisite area of research for the Wayamba UUV.

To assess potential operational capabilities and optimise concepts of operation for unmanned maritime systems, a real-time interactive simulation tool based on game engine technology is currently under development. This includes developing interfaces to allow external simulations to control the state of internal game engine objects. The aim of this work is to combine the sophisticated visualisation capabilities of game engines with the high fidelity simulation results that are available through the use of packages such as seakeeping codes.

\section{CONCLUSIONS}

This work outlines the results of research undertaken at DSTO into the development of a virtual simulation capability for the Wayamba UUV. Key components of this simulation suite were outlined, including a low-level model for investigating vehicle dynamics and performance and higher-level COTS UUV software capable of providing hardware-in-the-loop simulation capabilities. The representativeness of these models was demonstrated by comparing simulation results with real vehicle data obtained from field trials. In order to further develop and improve the fidelity of this simulation capability, outstanding areas of research and directions for future work were also provided, with an emphasis on including the through body thrusters into the low-level model. These improvements will increase the reliability of predictions about how vehicle design changes impact upon its manoeuvrability and allow the models to be used in more detailed simulations.

\section{ACKNOWLEDGEMENTS}

The authors gratefully acknowledge the support and guidance provided by DSTO's Maritime Division and the trials support provided by colleagues within the Automation \& Unmanned Maritime Systems Group. The lowlevel vehicle model was initially developed by Kariza Martin as part of a DSTO summer vacation scholarship program. MATLAB ${ }^{\circledR}$ figures were converted using Nico Schlömer's mat lab2tikz script.

\section{REFERENCES}

Australian Department of Defence (2013). 2013 Defence white paper.

Coxhead, M., P. Graham, R. Neill, P. Price, A. Travers, J. Wharington, and G. Wright (2002, October). A report card on Wayamba, DSTO's new uninhabited undersea research vehicle. In Proceedings of the International Conference on Undersea Defence Technology (UDT Korea 2002), Cheju, Korea, pp. 1-10.

de Barros, E., J. Dantas, A. Pascoal, and E. de Sa (2008). Investigation of normal force and moment coefficients for an AUV at nonlinear angle of attack and sideslip range. IEEE Journal of Oceanic Engineering 33(4), 538-549.

Evans, J. and M. Nahon (2004). Dynamics modeling and performance evaluation of an autonomous underwater vehicle. Ocean Engineering 31(14), 1835-1858.

Fossen, T. (1994). Guidance and Control of Ocean Vehicles. New York, USA: John Wiley \& Sons.

H-Scientific (2010). AUVSim 3 user manual.

Healey, A. and D. Lienard (1993). Multivariable sliding-mode control for autonomous diving and steering of unmanned underwater vehicles. IEEE Journal of Oceanic Engineering 18(3), 327-339.

Madden, C., J. Gilbert, and B. Knox (2012). Integrating the spectre autopilot into the wayamba research platform. DSTO Report DSTO-TN-1124, Defence Science and Technology Organisation.

Nahon, M. (1996). A simplified dynamics model for autonomous underwater vehicles. In Proceedings of the 1996 Symposium on Autonomous Underwater Vehicle Technology (AUV'96), Monterey, CA, pp. 373-379.

Schnoor, R. T. (2003, September). Modularized unmanned vehicle packages for the littoral combat ship mine countermeasures missions. In Proceedings of MTS/IEEE OCEANS 2003, San Diego, USA, pp. 1437-1439. 